\title{
Gasimo: A Global Address Space Simulation Model
}

\section{(Poster Abstract)}

\author{
Worawan Marurngsith \\ Department of Computer Science, \\ Faculty of Science and Technology, \\ Thammasat University, Pathum Thani, 12121 Thailand \\ +6629869157, +66 813444037 \\ wmrs@cs.tu.ac.th
}

\author{
Roland N. Ibbett \\ Edinburgh Parallel Computing Centre, \\ University of Edinburgh, \\ Edinburgh EH9 3JZ UK \\ +441316505030 \\ R.N.lbbett@ed.ac.uk
}

\begin{abstract}
The partitioned global address space (PGAS) programming model has gained attention as a robust model suitable for a diversity of emerging concurrent architectures. PGAS offers more scalability over the former distributed shared memory system (DSM) by supporting asynchronous execution based on message passing. Combining asynchronous communication with the facility to make the location of data transparent, applications written in PGAS languages have to trade off the benefits of concurrent architectures with the overhead caused by accessing distant memories.

Here we present an effective simulation model to reflect the cost of distant memory accesses on a PGAS system. The model, called Gasimo, simulates a generic PGAS execution environment on top of a cluster of homogeneous dual-core machines. Gasimo is a parallel extension of a particular DSM simulator, called DSiMCluster, which has been implemented on top of a discrete event simulation (DES) engine known as HASE.
\end{abstract}

\section{Categories and Subject Descriptors}

I.6.5 [Simulation and Modeling]: Model Development Modeling methodologies; I.6.8 [Simulation and Modeling]: Types of Simulation - Parallel; C.1.2 [Processor Architectures]: Multiple Data Stream Architectures (Multiprocessors) - Multipleinstruction-stream, multiple-data-stream processors (MIMD).

\section{General Terms}

Design, Performance, Experimentation, Verification

\section{Keywords}

Parallel discrete-event simulation (PDES), Multi-core, OpenMP, Partitioned Global address space (PGAS), Simulation Model

\section{INTRODUCTION}

The emergence of concurrent architectures such as clusters of symmetric multiprocessors, heterogeneous accelerators, large core-count integrated machines, and multithreaded multi-core machines have created a demand for a suitable, robust programming model such as a partitioned global address space (PGAS). Similar to the concept of distributed shared memory systems (DSM), PGAS allows applications to access data in a

Permission to make digital or hard copies of all or part of this work for personal or classroom use is granted without fee provided that copies are not made or distributed for profit or commercial advantage and that copies bear this notice and the full citation on the first page. To copy otherwise, or republish, to post on servers or to redistribute to lists, requires prior specific permission and/or a fee.

SIMUTools 2010 March 15-19, Torremolinos, Malaga, Spain.

Copyright 2010 ICST, ISBN 78-963-9799-87-5. logically shared address space by abstracting away the distinction of physical memory location. PGAS offers more scalability over the DSM by supporting asynchronous execution using message passing. Combining asynchronous communication with the facility to make the location of data transparent, applications written in PGAS languages have to trade off the benefits of concurrent architectures with the overhead caused by accessing distant memories [1]. This creates a requirement for an efficient tool to analyse such performance tradeoffs.

Current research attempts to simulate a diversity of concurrent architectures such as a simulation of clusters of symmetric multiprocessors [2], simulation of heterogeneous accelerators [3], simulation of large core-count integrated machines [4] and simulation of multithreaded multi-core machines [5]. However, a model which could reflect the performance tradeoffs of the PGAS system is not yet available.

Here we propose Gasimo, a simulation model of a PGAS system on top of a cluster of homogeneous dual-core machines. The proposed model is a parallel extension of a particular DSM simulator, DSiMCluster, which has been implemented on top of a discrete event simulation (DES) engine known as HASE.

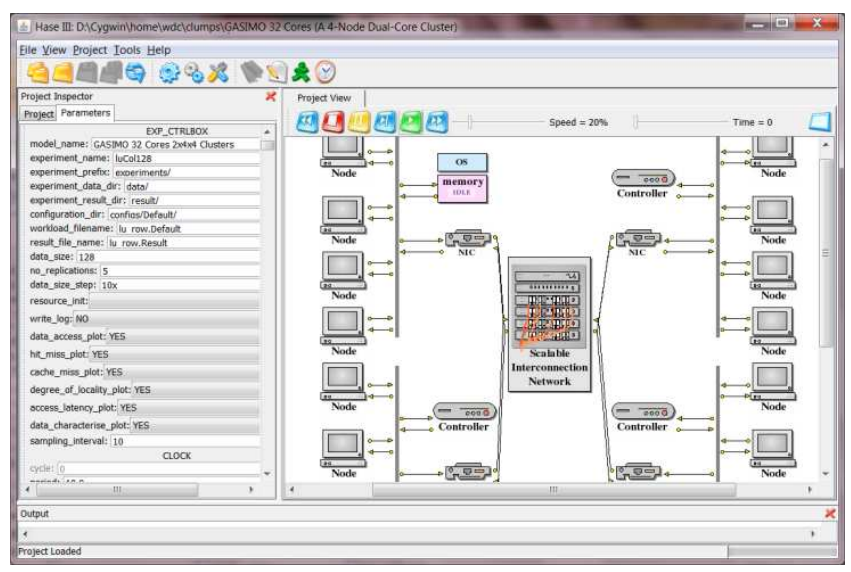

Figure 1 The Gasimo 4x4 Dual-Core Cluster Model

\section{THE GASIMO MODEL}

Gasimo is a parallel extension of a DSM Simulator called DSiMCluster [2] built on top of HASE ${ }^{1}$, a legacy DES simulation framework. The model has been developed on Windows 7 with Intel Software Tools ${ }^{2}$ and integrated with the HASE-III environment as shown in Figure 1.

\footnotetext{
1 http://www.icsa.inf.ed.ac.uk/research/groups/hase/

2 http://software.intel.com/en-us/intel-sdp-home/
} 


\subsection{HASE Framework and DSiMCluster}

The Gasimo model is built on the DES simulation framework named HASE, a Hierarchical computer Architecture design and Simulation Environment. HASE provides some mechanisms to support the following four steps in modeling a simulation: (1) model design, (2) construction of a simulation executable, (3) experimental control to set parameters and run a simulation and (4) tracing and post-mortem animation. Several research and teaching models have been implemented using HASE.

DSiMCluster is an reconfigurable model emulating a system with multiple shared caches with hierarchical coherence such as clusters of distributed shared memory systems. The simulator comprises an on-the-fly verification and has been shown to give a correct reflection of memory characteristics [6]. DSiMCluster is a sequential simulation model that runs parallel workloads by multithread interleaving to emulate a multithreaded runtime environment.

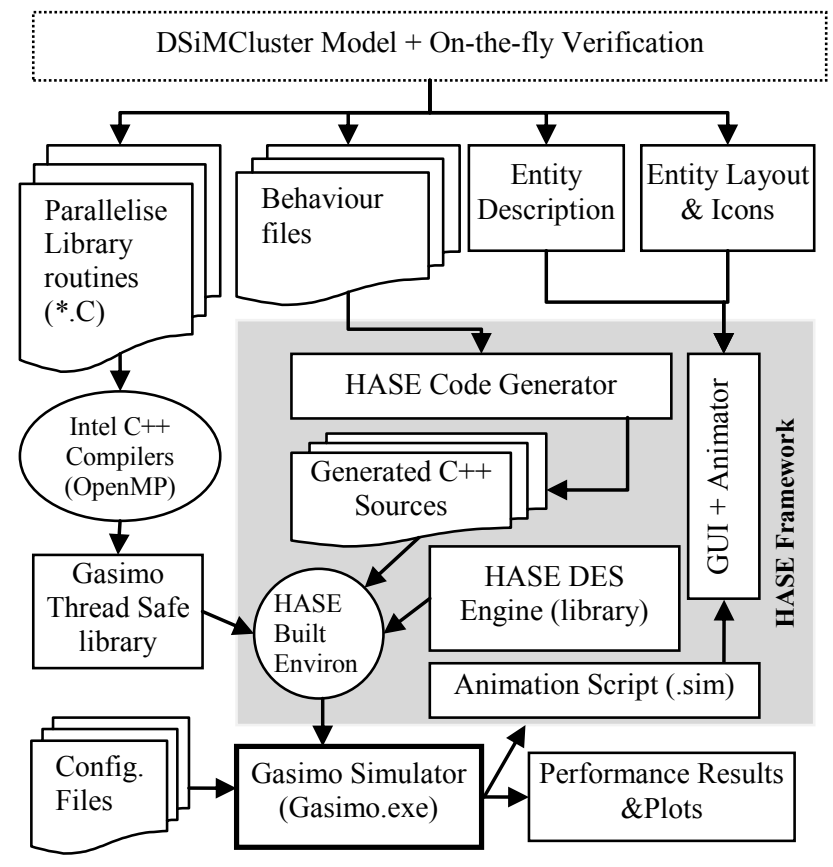

Figure 2 Gasimo Software Architecture

\subsection{Parallel extension in Gasimo}

Despite the extensibility of the DSiMCluster, its sequential implementation does not exploit the parallelism of recent multicore host machine. To address this limitation, we implement Gasimo by parallelising DSiMCluster in three parts. First, the main search loops in DSiMCluster library routines including the instruction set emulation module, cache controller and translation look-aside buffer have been parallelized using OpenMP, a standard shared memory programming model. Second, the behavior file implementing the processor entity has been modified to include OpenMP constructs in order to simulate a homogeneous dual-core processor. Two uni-processors are composed in a compound entity. Each compound entity is implemented as two parallel threads working in different functions (using OpenMP Sections). This is to emulate multiple instruction streams, multiple data stream (MIMD) execution. Third, the description and layout of the system entities include the new coupled processor entities. Figure 2 depicts the steps of the Gasimo extension and its integration into the HASE framework.

\section{Gasimo 4x4 Dual-Core Cluster Model}

We have tested the correctness of the Gasimo model by running a test program (LU) written in MPI and OpenMP, using 4 processes each of which creates 8 threads. Gasimo has been configured to represent a cluster of symmetric multiprocessor (SMP) machines consisting of 32 cores (see Table 1). Each SMP is an eight-core machine made up of four dual-core processors sharing the same physical memory. Four SMPs networked together on a single bus and were made into a single system by using the page-based global address space technique. Our preliminary test results show that the model can produce the correct output.

Table 1. Gasimo configurations

\begin{tabular}{|c|c|c|}
\hline Component & Variety & $\begin{array}{c}\text { Clock } \\
\text { Ratio }\end{array}$ \\
\hline $\begin{array}{c}\text { Processing } \\
\text { Elements }\end{array}$ & $\begin{array}{c}\text { 4x4 Dual-Core Cluster, an SMP } \\
\text { node comprises four dual core } \\
\text { processors (x86, 2GHz) }\end{array}$ & 1 \\
\hline \multirow{2}{*}{ Cache } & $\begin{array}{c}\text { Private L1, 64-Byte Block, Indexed } \\
\text { and Tagged using Virtual Address }\end{array}$ & 1 \\
\cline { 2 - 3 } & $\begin{array}{c}\text { Shared L2, 128-Byte Block, Address } \\
\text { Translation using 128 Bytes TLB }\end{array}$ & 2 \\
\hline \multirow{2}{*}{ Main Memory } & $\begin{array}{c}\text { 18-bit* Physical Address, } \\
\text { 32-bit* Virtual Address } \\
\text { (*half length of real machine) }\end{array}$ & 20 \\
\hline $\begin{array}{c}\text { Virtual Shared } \\
\text { Memory }\end{array}$ & $\begin{array}{c}\text { Page-based, 8KB Page Size, Lazy } \\
\text { Release Consistency Model }\end{array}$ & 1000 \\
\hline $\begin{array}{c}\text { Cluster } \\
\text { Interconnection }\end{array}$ & $\begin{array}{c}\text { Intra-node Bus } \\
\text { Inter-node Bus }\end{array}$ & $10^{4}$ \\
\hline
\end{tabular}

\section{FUTURE WORKS}

We are verifying and evaluating the model against measurement results. After verification, we plan to carry out memory analysis experiments on Gasimo using legacy benchmarks.

\section{REFERENCES}

[1] A. Kayi, et al., "Performance issues in emerging homogeneous multi-core architectures," Simulation Modelling Practice and Theory, vol. 17, pp. 1485-1499, 2009.

[2] W. Marurngsith and R. N. Ibbett, "DSiMCluster: A Simulation Model for Efficient Memory Analysis Experiments of DSM Clusters," SIMULATION, vol. 85, pp. 355-374, June 1, 2009.

[3] A. Bakhoda, et al., "Analyzing CUDA workloads using a detailed GPU simulator," in ISPASS 2009. pp. 163-174.

[4] R. Buyya, et al., "Modeling and simulation of scalable Cloud computing environments and the CloudSim toolkit: Challenges and opportunities," in High Performance Computing \& Simulation, 2009. International Conference on, 2009, pp. 1-11.

[5] G. H. Loh, et al., "Zesto: A cycle-level simulator for highly detailed microarchitecture exploration," in ISPASS 2009.

[6] W. Marurngsith and R. N. Ibbett, "Specification-based Verification in a Distributed Shared Memory Simulation Model," SIMULATION, p. 0037549709349843 , October 22, 2009. 